\title{
Feynman's figurations
}

\section{If a picture is worth a thous and words, a diagram can be worth many lines of complic ated algebraic formulas. Feynman diagrams are also pow erful tools of explanation and prediction.}

\section{Martin Kemp}

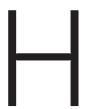
ow are we to understand Richard Feynman's statement, repeated in various forms, that "nobody understands quantum mechanics"? As the great authority on quantum electrodynamics and an eloquent communicator of difficult physics to nonspecialist audiences, his aphorism cannot be taken at face value.

He could not have meant that it was impossible to comprehend the basic principles, nor was he intending to dismiss the analytical and predictive efficacy of the mathematics of quantum mechanics. Rather, his assertion has to be interpreted in the light of the gravitational pull of physical modelling and graphic picturing in his approach to the physics.

He was continually concerned about the way that physical behaviour could be expressed in algebraic conventions without concrete visualization: "Strange! I don't understand how it is that we can write mathematical expressions and calculate what the thing is going to do without being able to picture it."

As Freeman Dyson recalled, "he was a natural physicist, and he thought in terms of concrete objects. The mathematics was an encumbrance, something you had to stick on afterwards, more or less ... as a necessary evil."

The now ubiquitous 'Feynman diagrams' achieved the graphic encoding of phenomena whose very nature means that they cannot be subject to direct picturing. In the 1949 paper that inaugurated his method, he provided the classic demonstration of the "fundamental interaction" in which electrons exchange a proton. The electrons are figured as solid lines, while the process of exchange of a 'virtual quantum' is represented by a wavy line, which graphically signals that the photon can be emitted from one electron and absorbed by the other, or if it travels backwards in time, in reverse. Either way, the result is the deflection of both electrons, denoted by their angular deviation.

Feynman diagrams look superficially like the simple graphics that physicists have used for centuries. But they are devices of exceptional power. Within their space-time coordinates, Feynman was able to sidestep the long-winded algebraic formulas that treated electrons and positrons separately. All the equations came together in one picture in a way that preceded and even directed calculation.

The diagrams rapidly and economically

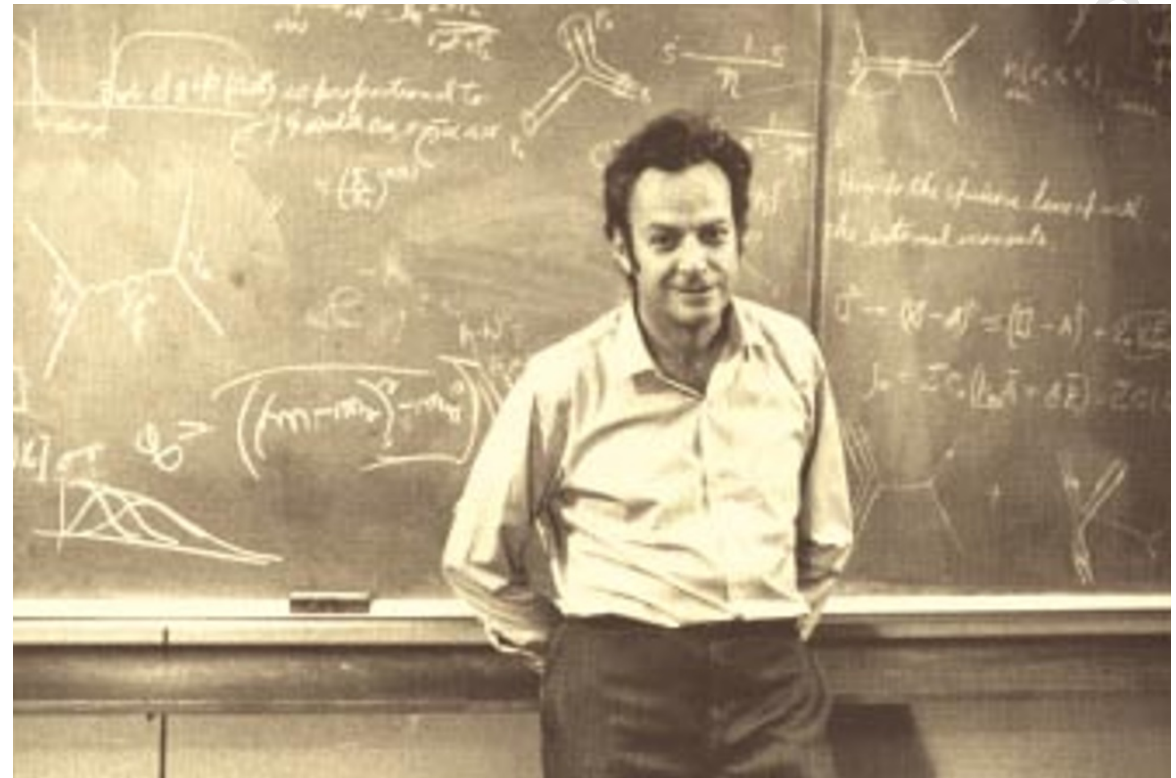

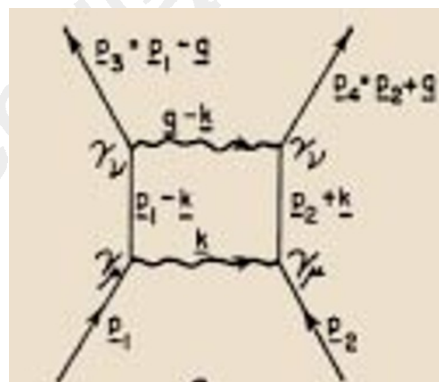

a.

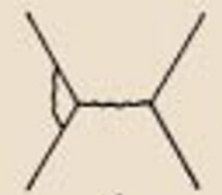

d.

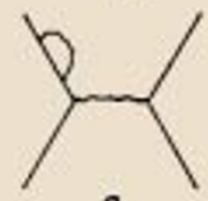

g.

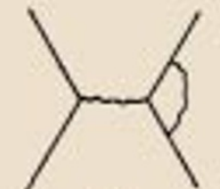

e.

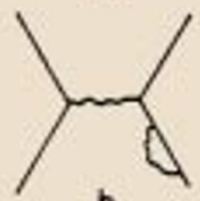

h.

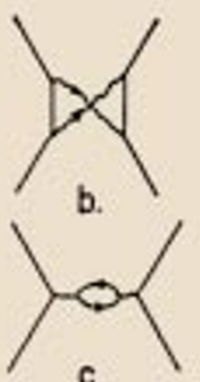

c.

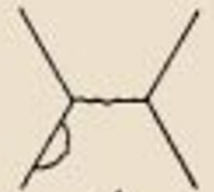

$f$

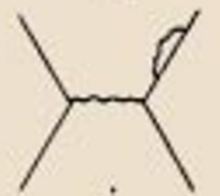

i
Feynman and his diagrams (from No Ordinary Genius: The Illustrated Richard Feynman, edited by Christopher Sykes; Weidenfeld and Nicolson, 1994).

Feynman diagrams of "the interaction between two electrons" (from "Space-Time Approach to Quantum Electrodynamics", Physical Review 76, 769-789; 1949).

explained and predicted in ways that were both intuitive and analytical. Feynman himself described the intuitive element in a characteristically graphic way: "It's like asking a centipede which leg comes after which — it happens quickly, and I'm not exactly sure what flashes and things go on in the head. I do know it's a crazy mixture of partially solved equations and some kind of visual picture of what the equation is saying is happening, but not as well separated as the words I'm using."

The diagrams mirror his conviction that "there is ... a rhythm and a pattern between the phenomena of nature $w$ hic $h$ is not apparent to the eye, but only to the eye of analys is".

Such a potent grammar of diagrams and matching equations provides a marvellous tool. Yet is it reasonable to wonder whether it also constrains what minds lesser than Feynman's permit themselves to envisage?

Martin Kemp is in the Department of the History of Art, University of Oxford, 35 Beaumont Street, Oxford OX12PG, UK. 